\title{
Electron ptychographic phase imaging using fast pixelated detectors
}

Hao Yang ${ }^{1}$, Roberto dos Reis ${ }^{1}$, Gerardo Martinez ${ }^{2}$, Lewys Jones ${ }^{2}$, Martin Simson ${ }^{3}$, Heike Soltau ${ }^{3}$, Yukihito Kondo ${ }^{4}$, Ryusuke Sagawa ${ }^{4}$, Timothy Pennycook ${ }^{5}$, Peter Nellist ${ }^{2}$

${ }^{1}$ Lawrence Berkeley National Lab, Berkeley, United States, ${ }^{2}$ University of Oxford, Oxford, United Kingdom, ${ }^{3}$ PNDetector GmbH, München, Germany, ${ }^{4}$ JEOL Ltd, Tokyo, Japan, ${ }^{5}$ University of Vienna, Vienna, Austria

E-mail: haoyang.tem@gmail.com

In the scanning transmission electron microscope (STEM), the commonly-used imaging modes, for example annular darkfield (ADF) or annular bright-field (ABF), form images by using detectors that integrate only a portion of diffraction intensities in the detector plane. Although such integrating detectors used for ADF and $A B F$ are able to respond at the timescales required for typical STEM scanning speeds, much of the wealth of information that is present as fine details in the electron diffraction intensity is unavoidably lost. Recent development in detector technology have resulted in cameras with frame-speeds that can exceed $1 \mathrm{kHz}$, and can therefore be used to record a so-called "four dimensional" (4D) data-set, which consists of a 2D diffraction pattern for each probe position of a 2D STEM scan, without inordinate increase in dwell time. Here we explore how the rich diffraction information in the recorded 4D data-set can be extracted efficiently to extend the capabilities of STEM to unlock new applications.

It was shown nearly 20 years ago that ptychography could be used to extract phase information from diffraction patterns that exceeded the conventional diffraction limit of the microscope used [1]. Here we make use of ptychography to form a quantitative image of the phase shift of the electron wave due to the sample, and to use those alongside the simultaneously formed ADF image to characterize the sample, see Figure. We compare the signal to noise ratio of ptychographic phase as a function of electron dose with a range of other phase imaging approaches, including differential phase contrast STEM and conventional HRTEM approaches [2].

We have applied the ptychography approach to a range of materials types. The combination of simultaneous phase imaging and Z-contrast is used to solve the previously unknown structure of a complex nanostructure [3]. We show that residual lens aberrations can be detected and corrected post-acquisition, and that 3D information can be reconstructed from a single tilt. We also show various examples of imaging light elements in solid state materials, and examine the robustness of the ptychography approach to dynamical electron diffraction.

[1] P.D. Nellist, B.C. McCallum, J.M. Rodenburg. (1995). Nature, 374, 630-632.

[2] T.J. Pennycook et al. (2015). Ultramicroscopy, 151, 160-167.

[3] H. Yang et al. (2016). Nature Communications. 7, 12532.

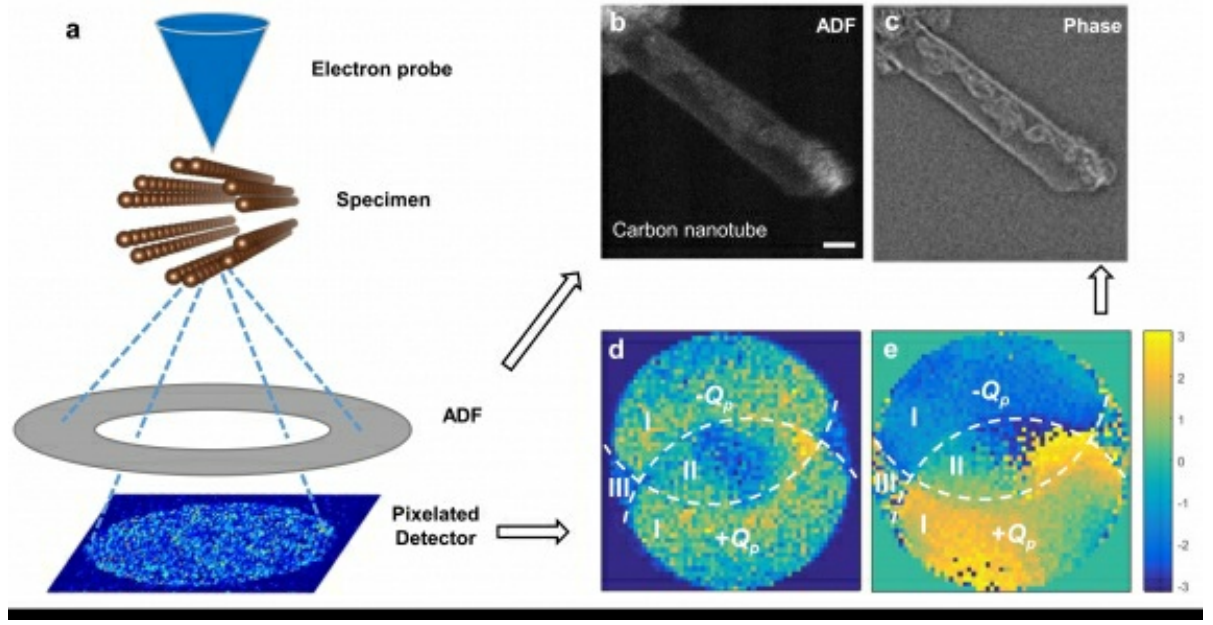

Keywords: ptychography, phase retrieval, STEM 